\title{
Overview of Multi-Agent Approach for Micro-Grid Energy Management
}

\author{
Meena Agrawal ${ }^{1}$, Arvind Mittal $^{2}$ \\ ${ }^{1,2}$ Department of Energy, Maulana Azad National Institute of Technology(MANIT), Bhopal, INDIA
}

\begin{abstract}
As the power generation paradigm is going green by involvement of renewable energy sources, the energy management process also has to evolve towards new more powerful, intelligent control methods. The inter-connection of a large number of renewable energy sources to the grid, along with their obvious advantages, makes the grid highly distributed. This may cause problems of efficient control, stability, reliability, power quality and security, thus represent one of the greatest engineering challenges. To enable greater amounts of distributed and renewable energy resources across the power grid backbone for providing better demand management, the next-generation electricity grid is emerging as a system of organically integrated intelligent Micro-Grids. In order to make them intelligent the application of Information \& Communication Technology (ICT), remote sensing and communication technology to power systems is gaining momentum making Micro-Grids smart. Agent-oriented software engineering paradigm represents an interesting means of analyzing, designing and building complex software systems, quite suitable to the requirements of making Micro-Grids smart. Multi-Agent System (MAS) seems to have marvelous features that meet the operation and control requirements of distributed energy resources in Micro-Grids. This paper presents an overview of the emerging sub-field of Distributed Artificial Intelligence, Multi-Agent System to control and manage the changing face of electric power grid by inculcating the intelligent agents into Micro-Grids.
\end{abstract}

Keywords: Distributed Renewable Energy Resources, Energy Management, Intelligent Agents, Micro Grid, Multi-Agent systems.

\section{INTRODUCTION}

Today's existing power grid is a hierarchical system, a one-way pipeline where the source has no realtime information about the service parameters of the termination points. The grid is therefore over-engineered to withstand maximum anticipated peak demand across its aggregated load and since this peak demand is an infrequent occurrence, the system is inherently inefficient. The next-generation power grid, known as the "Smart-Grid" being a convergence of ICT, remote sensing and power system engineering to allow consistent control and monitoring, is expected to address the major shortcomings of the existing grid. The smart grid is expected to be materialized as a well-planned plug-and-play integration of smart Micro-Grids that will be interconnected through dedicated ICT highways for command, data, and power exchange [1].

The National Science Foundation, Official department of the U.S Government has been funding a program and working jointly with Clemson University, Georgia Tech and Missouri on a 5 year project. The project is growing neurons in Petri dishes to control the Smart Grid. The Director of the Real-Time Power and Intelligent Systems Laboratory at Clemson University says-"To get the most out of the different types of renewable energy sources, we need an intelligent grid that can perform real-time dispatch and manage optimally available energy storage systems; What we need is a system that can take signals from a simulated power grid monitor, forecast, plan, learn and make decisions as well" [2]. The goal is to make the control systems more brain-like for Neuro-control of the power grid with new architectures and algorithms to carry out real-time control of complex systems.

Artificial Intelligence in Power Grids paves one such way, where Agent oriented software engineering paradigm represents an interesting means of analyzing, designing and building complex software systems, which are quite suitable to the new smart grid requirements. Agent oriented approach which is built around a new type of abstraction - an agent, seems to reshape the way, new information systems are designed \& developed. The agents interact with one another in order to realize their goal, thus forming a Multi-Agent society. The idea of Multi-Agent System MAS as a new concept of information systems' architecture appeared a couple of decades ago. Since then it has been utilized for numerous purposes in the field of robotics, planning, process control, communication networks, concurrent systems, artificial intelligence, distributed information systems and many others. In recent years Multi-Agent systems have gained tremendous popularity in providing solution to the distributed decision making problems in different domains of power system engineering. Scope of MAS applications study has spread to power systems restoration, normal operation, protection, control, monitoring, and also to maintenance scheduling, and electric power market simulation [3]. MAS applications cover a variety of domains, including aircraft maintenance, electronic book buying coalitions, wireless 
collaboration and communications, military logistics planning, industrial joint mission planning, financial portfolio management, robotics network security, computer games, e-commerce etc [4]. The unique features of Multi-Agent systems, autonomy, redundancy, robustness, and artificial learning, make it an interesting substitute for traditional software systems in the concerned application domains. McArthur et al. [5] and Funabashi et al. [6] explain that MAS have been applied to several types of problems in power engineering: diagnostics, distributed control, modeling and simulation, protection, maintenance scheduling.

The current paper presents a Multi-agent approach to Micro-Grid operation. This Section I of the paper gives an introduction of how MAS are used as energy management tools on distributed control, and more precisely on energy management in Micro-Grids. Section II elaborates Micro-Grids concept. Section III gives information about MAS in general and their main applications. Section IV discusses the Agents, their environment to methodologies. Section V is a short discussion about existing and future MAS applications and challenges to micro-grid energy management. Finally, Section VI summarizes and concludes the paper.

\section{RENEWABLE ENERGY MICRO-GRID}

The Smart Grid emerges as a system of organically integrated smart Micro-Grids at the distribution end of the power system, which accommodate various renewable energy technologies. The Micro-Grids (MG) comprise low voltage (LV) distribution systems with integration of Distributed Generation resources such as photovoltaic, wind, bio-mass, bio-fuel and fuel cell together with Distributed storages, like flywheels, energy capacitors, batteries and Controllable Loads behaving as a coordinated entity networked by employing advanced power electronic conversion and control capabilities. The Micro-Grid enables high penetration of distributed generation without requiring re-design of the distribution system [7]. An important characteristic of the MicroGrid, when interconnected, it interacts with the main grid as one entity - absorbing or supplying power to the grid. Micro-Grids can provide improved electric service reliability and better power quality to end customers and can also benefit local utilities by providing dispatch able load for use, during peak power conditions or allowing system repairs without effecting customer loads [8]. A Micro-Grid enables small communities to take control of their energy use and reduce their carbon footprint through a new and innovative way of generating and managing electricity.

\subsection{Micro-Grid Operation \& Control}

Micro-Grid controls need to insure that it can connect to or isolate itself, from the grid in a rapid and seamless fashion. Also the voltage and frequency can be regulated, reactive and active powers can be independently controlled, and can meet the dynamic needs of the loads. During disturbances, the generation and corresponding loads can separate from the distribution system to isolate the Micro-Grid's load from the disturbance and thereby maintaining service without harming the transmission grid's integrity [9]. The MicroGrid must also be flexible enough for new micro-sources to be added to the system, as per the load demand without modification of existing equipment. Effective energy management is a key to achieve vital efficiency benefits by optimizing production and consumption of heat, gas and electricity in a Micro-Grid [10]. Energy Management System with intelligent overall control makes possible an efficient and flexible operation of the Micro-Grid network.

\subsection{Making Micro-Grids Intelligent}

A potential stepping-stone to a modern nationwide smart energy grid is the Micro-Grids combined with local energy generation, conditioning and storage system. It has a dose of "IT smarts" to enable "intelligence" for more efficient and dynamic management of both energy demand and on-site energy generation. A smart Micro-Grid is a grid in which various distributed energy technologies are combined with intelligent software to both monitor and manage energy supply and demand. ICT, Communication, Automation /Control must be planned and implemented in synergy to achieve optimal result [11].

\section{MULTI-AGENT SYSTEMS}

Recently intelligent Multi-Agents have become the focus of intense research in fields of Computer Science and Artificial Intelligence. To incorporate smart control for effectual energy management of distributed renewable energy sources Multi-Agent system (MAS) is one of the most exciting and the fastest growing domains as reflected in the noteworthy research contributions [12] The Multi-Agent System (MAS) is a promising technology to fulfill the requirements of intelligent energy control and management of Micro-Grids as a prominently emerging sub-field of Distributed Artificial Intelligence. Multi-Agents overlay a way to elaborate systems that are decentralized rather than centralized, emergent rather than planned, and concurrent rather than sequential; and this is largely what characterizes distributed energy generation system. A MAS is a combination of several autonomous and intelligent entities called agents working in collaboration to achieve the overall goal of a system. 


\subsection{Agents Concepts}

Several definitions of an agent in a Multi-Agent system have been cohabited in the past. Broadly researchers agree that the term "agent" denotes a software entity which is autonomous, proactive \& social, has its own execution environment and decision powers in order to meet its design objectives (Wooldridge, 2009). Ferber Jacques, 1999 [13] sketches the historical origins of MAS research and gives a minimal definition of an agent and of MAS, so that all branches of Multi-Agent research can accept it. An agent can be a physical or virtual entity that can act, perceive its environment in a partial way, communicate with others, and has skills to achieve its goals and tendencies. An agent receives information about a state of its environment, take actions which may alter that state and expresses preferences among the various possible states. Stated more simply, agents have their own control over their behavior and internal states in any possible environment. Nwana et al define agents in terms of three behavioral attributes, Autonomy, Co-operation and Learning. [14]. Autonomy refers to the principle that agents can operate on their own to meet their goals without the need for human guidance. A key element of autonomy is pro-activeness, i.e., the ability to take the initiative rather than acting simply in response to their environment. Co-operation with other agents is paramount, and is the reason for having multiple agents in the first place. In order to co-operate, agents need to possess a social ability, i.e., the ability to interact with other agents and possibly humans via some communication language. For agent systems to be truly smart, they would have to learn as they react and/or interact with their external environment [15]. This has been clearly shown as Venn diagram in Fig.1.

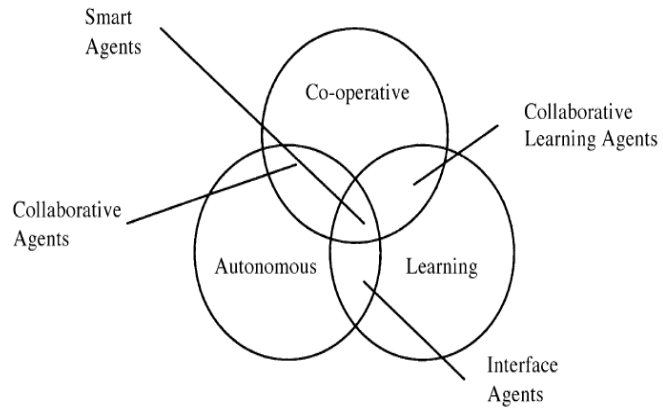

Fig:1 Agent Characteristics [15]

\subsection{Agent Frameworks}

Agent actions in the environment are typically mediated via an appropriate middleware or framework. This platform offers a design notion for Multi-Agent systems, providing means to govern resource access and agent coordination. In the context of object-oriented systems, an object offers the abstraction of a localized, encapsulated state characterized by a set of attributes and a publicly accessible set of services. In contrast to an object, besides a state and services a software agent has some additional capabilities: it has goals, autonomy, and usually an ability to cooperate with other agents. The addition of these simple features raise attractive possibility of software engineers performing high-level agent-oriented modeling and then being able to map them directly into complex, distributed information system implementations [17].

In order to support this kind of agent-oriented software engineering, there is a need for agent-oriented frameworks that fully support the agent modeling paradigm at multiple levels of abstraction. To define these interactions of multi agents, the Foundation for Intelligent Physical Agents, (FIPA) [18] proposes a platform, allowing agents to communicate with each other. The FIPA is a body for developing and setting computer software standards for heterogeneous and interacting agents and agent-based systems. FIPA was founded as a Swiss not-for-profit organization in 1996 with the ambitious goal of defining a full set of standards for both implementing systems within which agents could execute ie agent platforms and specifying how agents themselves should communicate and interact.

Many agent frameworks/ toolkits are available for designing and implementing MAS, like JANUS, JADE, ZEUS, Skeleton-Agent or Mad-Kit [19]. Most of these platforms offer functionalities that simplify the programming and use of a MAS which are useful for listing agents and their abilities. There are a variety of the FIPA implementations (ZEUS, RETSINA, FIPA-OS, and GRASSHOPER). One of them is Java Agent DEvelopment Framework, (JADE) [20] an open-source middleware used over the last years by academic and industrial organizations which is FIPA compliant. JADE, is a software framework for Multi-Agent systems in Java that has been in development over a decade. 


\subsection{Agents Environments}

The agents have their own discernment and analysis, which interact in a given environment. They sense input from the environment and generate an output that in turn affects the given environment as shown in the Fig. 2. The environment to agent reaction is a continuous process, whereby agents make out how their environment evolves and decide which actions are to be taken through their reasoning capability.

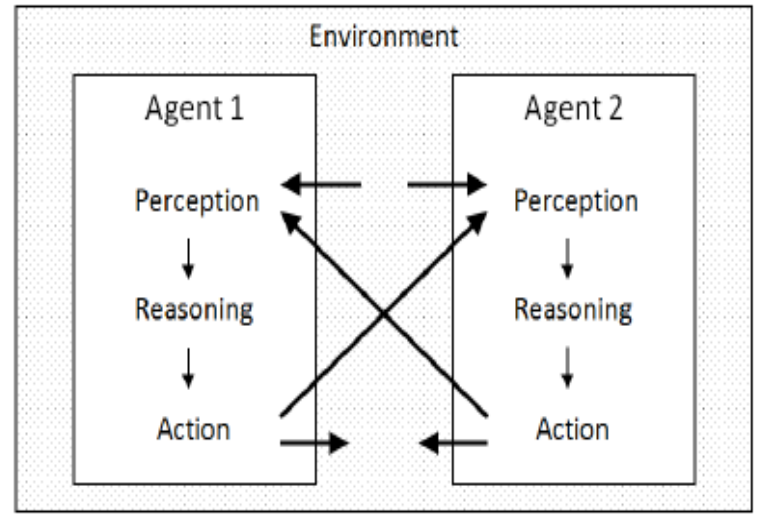

Fig.2. Agent Environments [16]

Agent Environment may be virtual, discrete or may be a continuous one. The separation between agents and their environment allows them to be shareable and thus used in very assorted kinds of situations. The intelligence of each agent is altered by a change of environment. Agents will react differently depending on their surroundings. The agents by nature are as such reactive and social which respond to environment changes or other agents' messages [16].

The inner strength of MAS is the ability of agents to interact with one another. Agents contain a list of well defined protocols that define methods of communication with different entities belonging to the system. An interesting analogy of MAS is a human group living among a society. Together by communicating with one another, all agents have a form of dispersed but cooperative intelligence. By its nature, agent technology makes possible the implementation of scalable, flexible and distributed systems. Thus MAS is able to address many of the problems of Micro-Grid energy management.

\subsection{Agent Communication}

Agents must have a common language so as to define how messages are written to communicate with each other. Such a language include some structure, some attributes corresponding to an agent. Agent communication is carried through message transfer whose depiction is based on the Agent Communication Language (ACL) or Knowledge Query and Manipulation Language (KQML) prepared by FIPA. To support the FIPA-based communication it is simply necessary to install a specialized agent that implements the FIPA Agent Communication Channel which will take care of transporting, marshalling, and encoding of messages. FIPA provides a standard for messaging and creating two directory concepts: an agent management service like white pages, a list of registered agents and a directory facilitator like yellow pages, a list of registered services [16]. FIPA does not specify any architectural restriction concerning the kind of communication that must take place within a given platform.

Y.Shoham proposes [21] that a fully developed Agent Oriented Programming (AOP) system is desired to have three components: a logical system for defining the mental state of agents; an interpreted language for programming agents; an 'agentification' process, for compiling agent programs into low-level executable systems.

To define the concepts and how agents relate to each other the type of vocabulary the messages use is to be identified This information is used to establish the agent's knowledge base, or Ontology. Ontology is the agents' knowledge base and vocabulary used by agents during communication. During the process of internal modeling, an agent's beliefs that represent its knowledge, and its plan that enables the agent to act autonomously are defined [22]. Belief is considered to be data that an agent possesses. It contains information about the environment and the agent itself which is updated on a continual basis.

\subsection{Agents Categorization}

For understanding and designing agents the use of taxonomy of agents is important, as the agents are there after defined by the combination of these qualities. Agents might be classified according to the duties they perform. e.g. information gathering agents or email filtering agents, or by the range and effectiveness of their 
actions, or by the degree of their internal state e.g., goal-driven agents to those with a full beliefs, desires and intentions reason capability.

In AI, an intelligent agent is an autonomous entity which observes through sensors and acts upon an environment using actuators and directs its activity towards achieving goals. Intelligent agents may also learn or use knowledge to achieve their goals. They may be very simple or very complex. Russell and Norvig, 2003 [23] group agents into five classes based on their degree of perceived intelligence and capability:

- Simple Reflex agents

- Model-based Reflex agents

- Model-based, Goal-based agents

- Learning agents

Ming Lu Zhu \& CL Philip chen [24] have noted the huge amount of work it takes to program an intelligent machine, and has concluded that it would be easier to build learning machines and then to teach them. Another advantage of learning agents is their adaptability to unknown environments, and the improvement of their behavior with time. Learning agents use the feedback from a critic to learn which perceptions of the environment are desirable, and in consequence, how to behave. Precisely, agents' learning consists in improving their future performance based on their past feedback from the critic, by optimizing their behavior such as to maximize their utility when the world continues evolving as it has been - Fig:3.

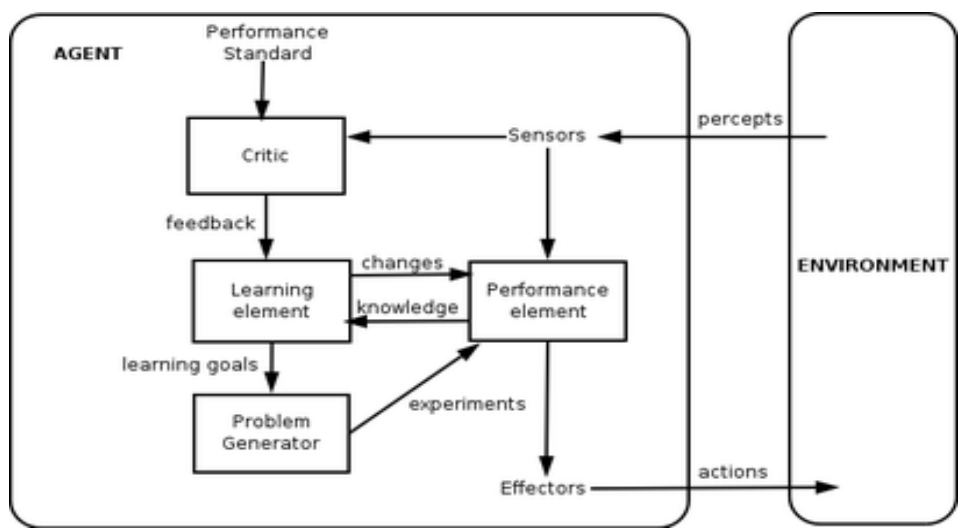

Fig:3 A general Learning agent [25]

\subsection{Agents Methodologies}

Specifying the system and its objectives carefully is essential in determining what its tasks are. From this the roles of agents can be defined and some models created. Establishing a systematic methodology is absolutely essential to utilize the full potential of agents. There have been quite a lot of attempts at creating tools for building agent-oriented software systems over past few years. A survey on currently available agent tools and methodologies has been presented by Tveit [26].

Currently there exist a number of agent-oriented methodologies such as Gaia, ROADMAP, Tropos, Prometheus, and MaSE. For analysis, design and development of agent-oriented systems each methodology provides a toolkit support also. Each methodology is focused on different type of agent applications, matching the methodology with the system requirement need further investigation. For instance, if we need belief agents, Prometheus is good; if the system has heterogeneous agents, then MaSE is the most suitable method; but for coarse grained computational agents, ROADMAP is recommended [27]. There have been various attempts at analyzing and comparing agent-oriented tools and methodologies to choose the most appropriate one. Yet one of the most elementary obstacles to large scale take-up of agent technology is the lack of mature agent-oriented software development methodologies.

\subsection{Agents Architectures}

Agents have a kind of intelligence of their own that gives them a certain degree of autonomy, depending on how they are structured or modeled. An agent interacts between external environments depending upon its own constitution cooperates with other agents depending upon its organization towards them and thus processes to solve problems. The MAS architecture focuses on two major aspects: agent organization architecture in view of agents' coordination, and agent's internal architecture in view of agent's autonomy. The former considers system's reliability and flexibility in terms of agents' interaction. The latter deals with each agent being able to respond to changes that occur in a dynamic environment based on its current state and its set of beliefs. Based on the analyzed goals, agents need to be identified and their relationships need to be modeled. For agent identification, link between goals and agents are to be examined [28]. 


\section{MAS MICRO-GRID ENERGY MANAGEMENT}

Conventional electrical power system has its own control and communication design consisting of various hardware and software protocols for exchanging system status \& control signals for the operation and overall management. This has been effectively achieved by Supervisory Control and Data Acquisition (SCADA) systems since years. In grid evolution by allowing a new renewable decentralized structure to exist, electricity generation will happen in much lower scales in terms of power and in much higher scales in terms of generator count. This indicates to manage a lot more generators with low power ratings like a large thermal plant could be replaced with several hundreds of solar panels or wind turbines. This progression will cause a major problem for managing the energy of so many generators and use it efficiently and reliably with even more loads. [29] For systems of this diversification the traditional control methods are not appropriate and other control system must be set up. It has become abundantly clear that large-scale penetration of renewable-based, distributed resources is impossible without attendant application of distributed storage and decentralized control.

As indicated in deliberations in earlier sections MAS have interesting characteristics that meet these requirements. As in MAS, contrary to classical analytical methods, the grid is considered to be a collection of simple entities ie agents - corresponding to sources, loads and other components, evolving in a given environment. A certain degree of distributed or collective intelligence can be achieved through the interaction of these agents with each other, cooperating or competing to reach their goals. MAS thus offer their inherent benefits of flexibility, extensibility, autonomy, reduced maintenance and much more for Micro-Grid energy management over the classical analytical control method SCADA system. The agent system also takes autonomous decisions such as seamless transition of Micro-Grids from grid-connected to island mode, loadshedding, securing critical loads etc. [30].

\subsection{Advantages of MAS in Micro-Grids}

MAS are flexible, plug \& play and fault-tolerant as a Micro-Grid is. MAS has the prospect to add new functionalities without having to entirely redesign the system. Hence they are well-suited for distributed problems. The constitution of MAS or of its environment can change without any considerable consequence for the functioning of the MAS itself. In the case of a grid, if a generator or a load is added or deleted that is, turned on or off, the MAS will acknowledge this modification and take it into account. This is a significant advancement in comparison to analytical control methods, where all possible events and changes have to be taken into account when designing the control system. Here, the MAS is capable of doing it by itself. Another interesting aspect of flexibility in MAS is fault tolerance which can be observed when a part of the MAS fails may be due to an inoperative component or a disrupted contact channel. A MAS is capable of continuing to work and trying to reach its goals even without some parts of its structure and can adapt to it [30].

The two previous characteristics enable this third one: MAS are well-suited for solving difficult problems, where the complexity can be distributed among several agents. The agents can operate autonomously if needed, and together as MAS at the same time, the complexity of the control system can be highly reduced by distributing it among communicating agents. Agents are not dependent on a physical support and can be located anywhere if they can communicate with the system. In fact, this is what MAS for a smart grid would do. The whole smart grid will be divided into Micro-Grids containing several generators, loads and storage devices. Intermediary layers consisting of groups of Micro-Grids may be added in between. The control of the grid is thus decentralized and decisions, like accepting or refusing to execute an action, can be taken locally. Unlike in today's control systems, a bottom-up approach is allowed to be used. In MAS each agent is individually motivated and attempts to maximize its own utility; each agent has incomplete information or capabilities for solving the problem, thus each agent has a limited viewpoint; data is decentralized; and computation is asynchronous [31]. Agents often co-operate to achieve their own individual goals, rather than to solve a common problem. Such approach offers a way to relax the constraints of centralized, planned, sequential control which is well the case of Micro-Grid energy management. MAS will increase real-time responsiveness to changing power loads \& component failures, improve the dynamic \& transient behavior of the power network, improve grid reliability, ensure local \& wide area stability, assist human experts in control rooms and reduce greenhouse gas emissions too.

\section{CONCLUSION}

This paper has presented an exhaustive overview of the research \& development of Multi-Agent systems (MAS) and its application possibilities as a solution to distributed hybrid renewable energy generation system. An introduction Micro-Grids and evolution smart grid are presented. To incorporate smart control for effectual energy management of Micro-Grids rapid emergent Multi-Agent system (MAS) technology is proposed. The concept of an agent, its environments, the notions, the attributes are deliberated. Research developments in agents frameworks, platforms as middle-wares, agent architecture and agent methodologies have been conversed. MAS architecture is not dependent on a particular technology, different programming 
languages can be used and interact together, if proper messaging tools are used. It identifies the key issues in the development and deployment of agent-based systems, and indicates how these issues should be addressed in the renewable energy resources Micro-Grids domain. It is observed that the field of agent-oriented methodologies and architecture is maturing rapidly and that the time has come for standardization of the next generation of agent-oriented software engineering methodologies. Efficient energy management being a necessary condition for making the Micro-Grids and hence the smart grid factual, MAS will certainly play an important role in the years to come.

\section{REFERENCES}

[1] H. Farhangi, The path to smart grid, IEEE Power and Energy Magazine, Jan/Feb 2010

[2] SmartGridNews.com,The National Science Foundation (NSF) Official department of U.S website.

[3] S. McArthur, E. Davidson, V. Catterson, A. Dimeas, N. Hatziargyriou, F. Ponci, and T. Funabashi, Multi-Agent Systems for Power Engineering Applications - Part I, Power Systems, IEEE Transactions, vol. 22, no. 42007

[4] Adhau, S., et al., A Multi-Agent system for distributed multi-project scheduling: An auction-based negotiation approach. Engineering Applications AI 2012:

[5] S. D. J. McArthur, E. M. Davidson, G. J. W. Dudgeon, and J. R. Mc- Donald, "Toward a model integration methodology for advanced applications in power engineering," IEEE Trans. Power Syst., vol. 18, no. 3, 2003

[6] T. Funabashi, T. Tanabe, T. Nagata, and R. Yokoyama, "An Autonomous Agent for Reliable Operation of Power Market and Systems Including Micro-Grids” IEEE 2008 Nanjing China

[7] Benjamin Kroposki, "An Integration Facility to Accelerate Deployment of Distributed Energy Resources in Micro-Grids" IEEE 2009

[8] B. Kroposki, R. Lasseter, T. Ise, S. Morozumi, S.Papatha-nassiou, and N. Hatziargyriou, "Micro-Grids: Technologies and Testing", IEEE Power and Energy Magazine, May 2008.

[9] J. A. Peças Lopes, "Defining Control Strategies for Micro-Grids Islanded Operation", IEEE,C. L. Moreira, and A. G.Madurei IEEE Trans on Power Systems, VOL.21, NO. 2, MAY 06.

[10] J. D. Kueck, R.H. Staunton, S. D. Labinov, B.J. Kir, "Micro-Grid Energy Management System" coordinated by CERTS, and Oak Ridge National Lab for U.S ,DoE, January 29, 2003

[11] Pipattanasomporn, M., Feroze, H., Rahman, S., "MAS in a Distributed Smart Grid: Design and Implementation", IEEE Power Systems Conference, Seattle, WA, USA, March 15-18, 2009

[12] Janeth G. Gómez-Gualdrón, Member, IEEE, Miguel Vélez-Reyes, Senior Member, Self-Reconfigurable Electric Power Distribution System using Multi-Agent Systems IEEE, 2007 IEEE.

[13] J. Ferber, Multi-Agent Systems: An Introduction to Artificial Intelligence. Addison-Wesley, 1999.

[14] H.S. Nwana, Software agents: an overview. Knowledge Engineering Review 113 (1996).

[15] H. Nwana, D. Ndumu, A perspective on software agents research. Knowledge Engg Review 1999.

[16] Jeremy Lagorse, Damien Paire, Abdellatif Miraoui; A Multi-Agent system for energy management of distributed power sources (Elsevier, France 2009).

[17] Michael Luck, Peter McBurney, and Chris Preist. Agent technology: Enabling next Generation computing, 2000

[18] Foundation for Intelligent Physical Agents. FIPA. http://www.fipa.org/spec/index.html. 2010

[19] JANUS Project website. www.janus-project.org

[20] Java agent development framework website www.jade.tilab.com

[21] Y. Shoham. Agent-oriented programming, Technical Report STAN-1335-90, Stanford University

[22] Z. Skolicki and T. Arciszewski, Intelligent agents in design. (2003).

[23] S. Russell and P. Norvig, Artificial Intelligence:Modern Approach.,( Prentice-Hall, London 1995).

[24] Mingzhu Lu C. L. Philip Chen, The design of Multi-Agent based distributed energy system. Proceedings of the 2009 IEEE international conference on Systems,

[25] Wikipedia Artificial Intelligence, Multi-Agent Systems.

[26] Tveit, A.: Survey of Agent-Oriented Software Engineering, First NTNU CSGSC 2001

[27] Ebrahim Al-Hashel, Bala M. Balachandran, and Dharmendra ; A Comparison of Three Agent-Oriented Software Development Methodologies: ROADMAP, Prometheus, and MaSE; University of Canberra, ACT 2601, Australia , Part III, LNAI, 2007.

[28] Sooyong Parka, Vijayan, Sugumaranb. Designing Multi-Agent systems: a framework and application, Expert Systems with Applications South Korea 2005, Sogang University, Seoul.

[29] S. Rahman, M. Pipattanasomporn and Y. Teklu, Intelligent Distributed Autonomous Power Systems (IDAPS), In Proc. 2007 the IEEE PES, Florida

[30] Robin Roche, Benjamin Blunier, Abdellatif Miraoui, Vincent Hilaire, Abder Koukam . Multi-Agent Systems For Grid Energy Management: University of Belfort, France, IEEE, 2010.

[31] Shehory, O., Sturm, A.: "Evaluation of modelling techniques for agent-oriented systems". Fifth International Conference on Autonomous Agents, 2001 